\title{
The effect of milk supplementation on bone mineral density in postmenopausal Chinese women in Malaysia
}

\begin{abstract}
Dietary studies often report low calcium intake amongst post-menopausal Malaysian women and calcium deficiency has been implicated as part of the etiology of age-related bone loss leading to osteoporosis. Therefore, the objective of this study was to examine the effectiveness of high calcium skimmed milk (Anlene Gold ${ }^{\mathrm{TM}}$, New Zealand Milk, Wellington, New Zealand) to reduce bone loss in Chinese postmenopausal women. Two hundred subjects aged 55-65 years and who were more than 5 years postmenopausal were randomized to a milk group and control group. The milk group consumed $50 \mathrm{~g}$ of high calcium skimmed milk powder daily, which contained $1200 \mathrm{mg}$ calcium (taken as two glasses of milk a day). The control group continued with their usual diet. Using repeated measures ANCOVA, the milk supplement was found to significantly reduce the percentage of bone loss at the total body compared to the control group at 24 months (control $-1.04 \%$, milk $-0.13 \% ; \mathrm{P}<0.001)$. At the lumbar spine, the percentage of bone loss in the control group was significantly higher $(-0.90 \%)$ when compared to the milk $(-0.13 \%)$ supplemented group at 24 months $(\mathrm{P}<0.05)$. Similarly, milk supplementation reduced the percentage of bone loss at the femoral neck (control $-1.21 \%$, milk 0.51\%) $(\mathrm{P}<0.01)$ and total hip (control $-2.17 \%$, milk $-0.50 \%)(\mathrm{P}<0.01)$. The supplemented group did not experience any significant weight gain over the 24 months. The serum 25-hydroxy vitamin D level improved significantly $(\mathrm{P}<0.01)$ from $69.1 \pm 16.1 \mathrm{nmol} / \mathrm{l}$ at baseline to $86.4 \pm 22.0 \mathrm{nmol} / 1$ at 24 months in the milk group. In conclusion, ingestion of high calcium skimmed milk was effective in reducing the rate of bone loss at clinically important lumbar spine and hip sites in postmenopausal Chinese women in Malaysia. Supplementing with milk had additional benefits of improving the serum 25-hydroxy vitamin D status of the subjects.
\end{abstract}

Keyword: Bone mineral density; Calcium; Milk supplementation; Parathyroid hormone; Postmenopausal Malaysian women; Serum 25-hydroxy vitamin D 